\title{
Cognitive Behavioural Therapy for Auditory Hallucinations: Effectiveness and Predictors of Outcome in a Specialist Clinic
}

\author{
Neil Thomas \\ Monash Alfred Psychiatry Research Centre and La Trobe University, Australia \\ Susan Rossell \\ Monash Alfred Psychiatry Research Centre and Swinburne University, Australia
}

John Farhall and Frances Shawyer

La Trobe University, Australia

David Castle

University of Melbourne, Australia

\begin{abstract}
Background: Cognitive behavioural therapy has been established as an effective treatment for residual psychotic symptoms but a substantial proportion of people do not benefit from this treatment. There has been little direct study of predictors of outcome, particularly in treatment targeting auditory hallucinations. Method: The Psychotic Symptom Rating Scales (PSYRATS) and Positive and Negative Syndrome Scale (PANSS) were administered pre- and post-therapy to 33 people with schizophrenia-related disorders receiving CBT for auditory hallucinations in a specialist clinic. Outcome was compared with pre-therapy measures of insight, beliefs about the origin of hallucinations, negative symptoms and cognitive disorganization. Results: There were significant improvements post-treatment on the PSYRATS and PANSS Positive and General Scales. Improvement on the PSYRATS was associated with lower levels of negative symptoms, but was unrelated to overall insight, delusional conviction regarding the origins of hallucinations, or levels of cognitive disorganization. Conclusions: Lack of insight and presence of formal thought disorder do not preclude effective cognitive-behavioural treatment of auditory hallucinations. There is a need to further understand why negative symptoms may present a barrier to therapy.
\end{abstract}

Keywords: Schizophrenia, psychosis, auditory hallucinations, cognitive behavioural therapy, predictors, insight, negative symptoms.

Reprint requests to Neil Thomas, Monash Alfred Psychiatry Research Centre, Level 1, Old Baker Building, The Alfred, Commercial Road, Melbourne, Victoria 3004, Australia. E-mail: neil.thomas@ monash.edu 


\section{Introduction}

Cognitive behavioural therapy (CBT) has become established as an evidence-based treatment for reducing the emotional and behavioural impact of medication-resistant psychotic phenomena such as hallucinations and delusions in schizophrenia (National Institute for Clinical Excellence, 2009; Wykes, Steel, Everitt and Tarrier, 2008). However, up to 50\% of people fail to respond to this therapy (Garety, Fowler and Kuipers, 2000). In order to facilitate the recovery of people who have ongoing distressing psychotic experiences there is a need to understand what the barriers are to benefiting from psychological treatment.

In spite of there being over 30 randomized controlled trials of CBT for psychosis (Wykes et al., 2008), there have been relatively few reports of predictors of outcome. Some trials have reported that people with more severe and longstanding experiences of psychosis are less likely to show improvement following CBT (Durham et al., 2005; Tarrier et al., 1998), but these factors were identified as applying to both CBT and control treatments, rather than specifically applying to CBT. Identification of these broad variables also has limited implications for how to improve therapy outcomes. Of more clinical importance are specific clinical characteristics that may predict therapy response. A study of a wide range of predictors was conducted as part of one of the earlier clinical trials of CBT for psychosis (Garety et al., 1997). This study's key finding was that post-treatment outcome was related to a pre-therapy measure of whether service users could acknowledge the possibility that they were mistaken in their delusional beliefs, and also to a dimension of insight relating to awareness of the social disability associated with psychosis. Other variables, including IQ, verbal fluency, and probabilistic reasoning, were found to be unrelated to outcome. Another more comprehensive examination of predictors of outcome was conducted on data from the Insight into Schizophrenia Trial (Turkington, Kingdon and Turner, 2002), which examined a brief course of six sessions of CBT for psychosis delivered by community psychiatric nurses; higher levels of delusional conviction and lower overall levels of insight predicted poorer outcome (Brabban, Tai and Turkington, 2009; Naeem, Kingdon and Turkington, 2008).

These findings, relating to the overlapping constructs of strong delusional conviction, and lack of insight into psychotic experiences as mentally generated, suggest that CBT for psychosis may be less successful when people hold rigid delusional explanations of their experiences. This has been interpreted as there being a need for a "chink of insight" to conduct belief modification with people experiencing the reality distortion inherent in psychosis (Garety et al., 2000). This has received support from a qualitative study of experiences of therapists delivering CBT for psychosis (McGowan, Lavender and Garety, 2005). However, a difficulty in interpreting these findings is that they have been from trials of CBT for persisting positive symptoms in general, which may have involved addressing either delusions, hallucinations, or both in combination. Consequently, it is unclear whether insight and belief conviction are important solely in the treatment of delusions or also when hallucinations are the focus of therapy. Indeed, if poor insight and high delusional conviction impede the process of belief modification, this might not be a barrier to successful therapy if applying more behavioural methods such as coping strategy enhancement (Tarrier et al., 1993). A further limitation of studies combining CBT for delusions and hallucinations is that measurement of outcome has been reliant upon the use of broad mental state measures such as the Positive and Negative Syndrome Scale (Kay, Opler and Lindenmayer, 1987), which may be relatively insensitive to treatment effects on the specific symptoms targeted by therapy. 
Together with heterogeneity arising from differences in service user presentation and more than one potential focus of therapy, this may lead to further specific predictor effects being obscured.

Another strategy in examining predictors of outcome is to study CBT delivered for a specific target symptom. Auditory hallucinations, typically experienced as hearing voices, are one of the most common such targets, and they represent a discrete phenomenon for which more focused measures are available. Examining CBT for voices allows the role of belief conviction and insight to be clarified with this particular symptom. Also, by examining therapy targeted at a specific phenomenon with a dedicated outcome measure, other sources of variability in outcome are reduced, which may highlight other predictor variables.

A starting point in examining predictors of outcome in CBT for voices is to examine the influence of other psychotic phenomena on outcome. The symptoms of schizophrenia have been conceptualized in terms of three statistically-derived symptom clusters, which relate to distortion of reality, disorganization and negative symptoms (Liddle, 1987). Reality distortion symptoms include hallucinations, together with delusions and lack of insight. It is typical for auditory hallucinations to be associated with delusional beliefs about the origins and identity of voices (Chadwick and Birchwood, 1994). It is possible that strong conviction in these beliefs may present a barrier, not only to modifying these beliefs themselves, but also to restructuring related beliefs about voice power and control over the experience (Chadwick and Birchwood, 1994; Trower et al., 2004). Similarly, lack of insight into the origins of voices as internally generated may make it more difficult to establish a rationale for a psychological treatment, which may hinder formation of a working alliance.

Disorganization most frequently manifests as formal thought disorder, which often involves the person's speech frequently slipping into loosely related or unrelated topics. This may present a problem in the process of therapy as the person may be hard to direct through Socratic dialogue, and may have difficulties following the thread of therapy.

Negative symptoms such as affective blunting, amotivation and poverty of thought may make it difficult for the therapist to establish a working alliance with the service user and for the service user to engage in the sessions and complete between-session exercises. Indeed, a non-significant trend has been observed for affective blunting to be associated with a poorer outcome in CBT for psychosis (Brabban et al., 2009), suggesting that further study of negative symptoms is warranted.

The present study provides an examination of these potential predictors of outcome in an open trial of CBT for auditory hallucinations within a specialist clinic.

\section{Method}

\section{Participants}

Participants were people seen in a specialist outpatient clinic providing CBT for auditory hallucinations to people referred by public mental health services, private psychiatrists and general practitioners alongside their routine care. Inclusion criteria were: (a) a diagnosis of a schizophrenia or schizoaffective disorder; (b) current auditory hallucinations in the form of voices, occurring at least once per week; (c) voices associated with significant subjective distress; (d) history of voices for at least one year; and (e) currently prescribed antipsychotic medication. Of the 33 participants, $21(64 \%)$ were male and the overall mean age was 
36.4 years (SD 8.99). Ten (30\%) were in paid or voluntary employment. Participants reported hearing voices for an average of 13.5 years ( $S D$ 8.23). All participants had already been trialled on at least two separate antipsychotics prior to the outset of therapy.

\section{Therapy}

Participants received weekly to fortnightly treatment sessions up to a maximum of 24 sessions, with a mean therapy length of 12.1 ( $S D$ 5.98) sessions. In all, 26 participants received therapy from a clinical psychologist with 7 years' experience of CBT for psychosis, and the remaining 7 participants received therapy from other psychologists under fortnightly supervision of the main therapist. Therapy was based upon the manual of Fowler, Garety and Kuipers (1995) and broader literature on the psychological treatment of auditory hallucinations (e.g. Chadwick and Birchwood, 1994; Morrison and Renton, 2001; Tarrier, 1992). This included the development of a formulation based upon assessment of voice phenomenology, other psychotic symptoms, antecedents of and responses to hearing voices, and appraisals of voices that may mediate distress. This formulation was used to guide treatment, incorporating elements of building an adaptive shared understanding of hallucinatory experience, coping enhancement, developing adaptive responses to voices, and modifying appraisals relating to distress through Socratic questioning and behavioural experiments.

\section{Measures}

The following therapist-administered measures were completed at the beginning and end of therapy:

The Psychotic Symptom Rating Scales (PSYRATS; Haddock, McCarron, Tarrier and Faragher, 1999) is an interview-based set of clinician rating scales, comprising 11 5-point items assessing different dimensions of voice experience, such as frequency, distress, impact upon functioning. The total score was used as the main outcome measure, and the beliefs about origins of hallucinations scale (item 5) was used to assess strength of belief that voices heard are of external origin. High scores on this item correspond to strong delusional conviction that voices are externally generated, and low scores correspond to complete belief that voices are internally generated. The inter-rater reliability of the PSYRATS is excellent, including for specific items, and it shows good test-retest reliability and validity (Haddock et al., 1999; Drake, Haddock, Tarrier, Bentall and Lewis, 2007).

Positive and Negative Syndrome Scale (PANSS; Kay et al., 1987) is an interview-based measure of positive and negative psychotic symptoms, and other symptoms associated with psychosis, on three scales (positive, negative and general), comprising 30 7-point items. The negative syndrome scale was used to index negative symptoms, and the cognitive disorganization item (P2) was used to assess formal thought disorder. The PANSS shows excellent inter-rater reliability and validity (Bell, Milstein, Beam-Goulet, Lysaker and Cicchetti, 1992; Kay, Opler and Lindenmayer, 1988).

The Schedule for the Assessment of Insight (SAI; David, Buchanan, Reed and Almeida, 1992) is a clinician rating scale assessing three dimensions of insight into illness (awareness of illness, treatment compliance, and relabelling psychotic experiences) with a total score of 0-14; it has good inter-rater reliability and validity (David et al., 1992). 
Table 1. Pre-treatment scores on measures and correlations with outcome

\begin{tabular}{lrccc}
\hline & $M$ & $S D$ & $r_{p b}$ & $p$ \\
\hline PSYRATS total & 29.82 & 4.12 & +.13 & .46 \\
PANSS positive symptoms & 16.58 & 5.82 & +.06 & .74 \\
PANSS negative symptoms & 12.67 & 7.69 & -.64 & $<.001$ \\
PANSS general symptoms & 27.67 & 6.49 & -.18 & .32 \\
Belief about origin of voices & 2.61 & 0.97 & -.11 & .55 \\
Schedule for Assessment of Insight & 10.82 & 3.09 & +.12 & .51 \\
Cognitive disorganization & 2.00 & 1.70 & $+.07^{\text {a }}$ & .71 \\
\hline
\end{tabular}

Notes: Positive correlations represent associations with better outcome. $n=33$.

${ }^{a}$ rank biserial correlation.

\section{Results}

Twenty-eight participants $(85 \%)$ remained in the study and completed post-treatment assessments. Mean scores on the main measures at pre-treatment are presented in Table 1. Scores on each of the scales used were normally distributed, with the exception of PANSS Negative, which was transformed using a logarithmic function for analyses, and cognitive disorganization for which non-parametric statistics have been used. Including all 33 participants in the analysis, assuming no change in those who dropped out, participants showed improvements on PSYRATS (mean change $-5.09,95 \% \mathrm{CI}-7.31$ to $-2.87, t(32)=$ 4.633, $p<.001$, Cohen's $d=1.24$ ), PANSS Positive (mean change $-2.79,95 \%$ CI -3.97 to $-1.61, t(32)=4.818, p<.001$, Cohen's $d=0.48$ ) and PANSS General (mean change $=$ $-2.67,95 \%$ CI -4.40 to -0.93$), t(32)=3.128, p=.004$, Cohen's $d=0.76)$ scales at the end of treatment. There was no significant post-treatment change on PANSS Negative (mean change $=-0.33,95 \% \mathrm{CI}-1.31$ to $+0.64, t(32)=0.030, p=.98$, Cohen's $d=0.02$ ).

Outcomes of therapy were dichotomized according to whether an improvement of five points or more was obtained on the PSYRATS Auditory Hallucinations Scale. This corresponds to reliable change calculated from test-retest reliability data on the PSYRATS (Drake et al., 2007). Drop-outs were initially included in this analysis in order to provide an estimate of whether therapy had been effective on an intention-to-treat basis, assuming that drop-outs had not benefited. According to this criterion, 16 (49\%) participants showed improvement. To provide a standard effect size estimate across different types of data, correlation coefficients were calculated for each predictor variable's association with outcome, using point-biserial coefficients for normally distributed variables, rank-biserial coefficient for cognitive disorganization, and phi coefficients for categorical predictors.

Outcome was not significantly related to $\operatorname{sex}\left(\Phi=-.28\right.$, Yates' $\left.\chi^{2}=1.483, p=.22\right)$, employment status $(\Phi=.28$, Fisher's exact $p=.14)$, or age $\left(r_{p b}=-.29, p=.11\right)$, but did show a trend to be associated with length of illness $\left(r_{p b}=-.33, p=.06\right)$, with better outcomes for a shorter duration of illness. Outcome was not related to whether participants were seen by the main therapist or another therapist $(\Phi=.21$, Fisher's exact $p=.40)$.

The correlations between hypothesized predictor variables and outcome are presented in Table 1. There was no association between outcome and either insight or conviction that hallucinations were externally generated. Examination of individual dimensions of insight on 
the SAI likewise revealed no statistically significant predictors of outcome. Similarly, there was no association between outcome and cognitive disorganization.

Conversely, overall negative symptoms showed a statistically significant correlation with outcome. Exploration of the association between individual PANSS negative scale items and outcome found that all items showed at least a trend $(p<.06)$ to be associated with outcome $\left(r_{r b}=.33\right.$ to .57$)$, with the strongest effect sizes for items N2 (emotional withdrawal, $r_{r b}=$ .57 ), N4 (passive apathetic social withdrawal, $r_{r b}=.46$ ) and N3 (poor rapport, $r_{r b}=.45$ ). Outcome was not correlated with depression (PANSS item G6).

Repeating the analysis including only the 28 participants who completed therapy found a similar pattern of results: of the hypothesized predictors, only negative symptoms correlated with outcome at a statistically significant level $\left(r_{p b}=-.60, p=.001\right)$. However, in this analysis, outcome also correlated with both age $\left(r_{p b}=-.41, p=.03\right)$, and length of illness $\left(r_{p b}=-.38, p=.05\right)$. When controlling for age, length of illness and the number of therapy sessions attended, negative symptoms remained a statistically significant predictor of outcome (partial $r_{p b}=-.43, p=.03$ ).

Data on medication doses pre- and post-treatment were available for 24 of the 28 completers, and was examined in order to consider medication changes as a potential confounding variable influencing outcome. Only one participant switched to a different antipsychotic during therapy, and in the group as a whole average chlorpromazine-equivalent doses did not change significantly from pre- to post-therapy (pre: $M=793.1 \mathrm{mg}, S D=$ $468.6 \mathrm{mg}$; post $M=768.1 \mathrm{mg}, S D=473.8 \mathrm{mg} ; t(23)=0.518, p=.61)$. Within-participant changes in dose were not significantly related to outcome $\left(r_{p b}=-.28, p=.18\right)$.

\section{Discussion}

The study observed post-treatment improvements in hallucination severity when auditory hallucinations are a specific target of psychological treatment. Whilst the study was uncontrolled, these improvements arose independent of medication changes in a sample with longstanding treatment-resistant symptoms. This adds to findings from initial case series (e.g. Chadwick and Birchwood, 1994) and trials (Haddock, Slade, Bentall, Reid and Faragher, 1998; Trower et al., 2004), suggesting CBT to be an effective treatment for auditory hallucinations. In contrast to many previous studies that have focused on a specific element of CBT for auditory hallucinations, such as promoting recognition that voices are internally generated (Haddock et al., 1998), or restructuring beliefs about voice power (Trower et al., 2004), this study examined a formulation-driven approach incorporating elements of these different approaches according to the person's presentation. Such an approach is widely advocated in treatment manuals (e.g. Fowler et al., 1995; Morrison, Renton, Dunn, Williams and Bentall, 2004), and is likely to be more reflective of the delivery of CBT for voices in service settings. Hence, although the content of therapy was less standardized, the study has good ecological validity, which is particularly useful in considering predictors of outcome relevant to routine provision of therapy.

When examining predictors of outcome, key factors that were hypothesized as barriers to effective therapy did not emerge as such, with findings suggesting that CBT can be effective with voice hearers in spite of lack of insight, high delusional conviction or cognitive disorganization. The finding that outcome was unrelated to insight and belief conviction was in contrast to previous findings related to delusions. Whilst working with delusions is heavily 
reliant upon belief modification, CBT for voices may incorporate a wider range of therapeutic interventions, including the enhancement of coping strategies (Farhall, Greenwood and Jackson, 2007; Tarrier et al., 1993) and promoting disengagement from voices (Haddock et al., 1998). In addition, cognitive restructuring need not target beliefs about whether voices are real or hallucinatory, and may work effectively within the person's explanatory model to target beliefs such as the voices' ability to harm the person, or the person's level of control over the experience (Trower et al., 2004). It seems possible that by having a number of avenues of intervention available, therapists were able to bypass rigidity in beliefs that voices were real. Indeed, some emerging developments in psychological interventions to promote acceptance of or alter relationships with voices do not involve belief modification (e.g. Gaudiano and Herbert, 2006; Hayward, Overton, Dorey and Denney, 2009; Shawyer, Farhall, Sims and Copolov, 2005). Further study is required to clarify how the therapy process may be influenced by the levels of insight and delusional conviction, and specifically whether these lead to different therapeutic strategies being employed. However, given that insight has been suggested as a predictor of outcome, an important implication of findings is that lack of insight may not be a specific barrier to effective treatment of auditory hallucinations, and at the present time should not be used to judge suitability for treatment when delusions are not the focus of therapy.

These considerations similarly apply to cognitive disorganization. Again, there may be ways in which therapists use alternative routes such as a focus on more behavioural methods when service users exhibit high levels of disorganized speech and thinking. It may also be that the cognitive elements of therapy are helpful in assisting the person to develop a clearer understanding of their psychotic experiences, which their disorganized thinking makes it hard to attain. Indeed, there is some evidence that levels of cognitive disorganization may reduce following CBT (Tarrier et al., 2001). Further study may also help clarify this.

In contrast, negative symptoms emerged as a strong pre-therapy predictor of outcome. This was above any effect on drop-out, indicated by this association being maintained when analysing only completers and when controlling for number of sessions. This effect also appeared to be independent of length of illness. These data are consistent with the finding of a trend for affective blunting to be associated with poorer outcome in a trial of brief CBT for psychosis (Brabban et al., 2009). It may be that negative symptoms are a barrier to treatment specific to hallucinations, although it would be important to verify this association in other studies. Further study might also determine in more detail the specific barriers that may arise in people with negative symptomatology. For example, whilst the reasons for this association cannot be determined from the current data, it is possible to speculate that aspects of the negative syndrome interfere with engagement in therapy and manifest in measurable factors such as rapport with the therapist, session attendance and completion of homework. This might lead to modifications of CBT to compensate for the presence of negative symptoms, such as use of more behavioural methods, or to parallel intervention that also addresses negative symptoms (e.g. Rector, Beck and Stolar, 2005). Alternatively, it might be that therapies based upon direct cognitive remediation (e.g. Favrod, Vianin, Pomini and Mast, 2006) and more intensive coaching in coping with voices may be more suited to this population than therapies reliant upon a primarily verbal therapy.

It should, however, be noted that the negative syndrome is not a unitary construct, and reflects a range of symptoms, including aspects of subjective emotional experience, affect, thinking and behaviour. Data from this study using the PANSS, which does not provide a 
fully comprehensive measure of negative symptomatology, were too limited to be able to clearly delineate the specific aspects of the negative syndrome most predictive of outcome. Furthermore, whilst often characterized as representing core deficit psychopathology associated with schizophrenia (e.g. Kirkpatrick, Buchanan, Ross and Carpenter, 2001), the negative syndrome has also been conceptualized as an adaptational response to living with psychosis (and the associated responses of others), which may be related to factors such as defeatism and anticipation of social exclusion (e.g. Rector et al., 2005). More precise measurement of such constructs in future study may be more informative of targets for improving the effectiveness of therapy.

Limitations of this study include the small sample size. This resulted in reduced power to consider the relative importance of age and length of illness in predicting outcome. However, there were no non-significant findings for the hypothesized clinical predictors with estimated effect sizes of $r=0.20$ or more, which reduces the likelihood that clinically meaningful effects for these variables were undetected due to lack of power. The study did not include a control therapy condition, so the possibility that pre-treatment negative symptoms predicted poorer spontaneous improvement as opposed to being a barrier specific to CBT cannot be ruled out. In the absence of a control condition, assessments could not be masked, and were completed by therapists, which may have introduced bias. Long-term follow-up was not conducted, so findings are specific to post-treatment outcome, and may not apply to the durability of therapy effects.

Whilst bearing these limitations in mind, our findings suggest that therapists should consider the possible impact of negative symptoms when addressing the specific therapeutic target of auditory hallucinations.

\section{Acknowledgements}

This research was conducted at the Mental Health Research Institute of Victoria, funded by the William Buckland Foundation. We would also like to acknowledge the support of the mental health services of Melbourne Health, Mercy Health and St Vincent's Hospital Melbourne.

\section{References}

Bell, M., Milstein, R., Beam-Goulet, J., Lysaker, P. and Cicchetti, D. (1992). The Positive and Negative Syndrome Scale and the Brief Psychiatric Rating Scale: reliability, comparability, and predictive validity. Journal of Nervous and Mental Disease, 180, 723-728.

Brabban, A., Tai, S. and Turkington, D. (2009). Predictors of outcome in brief cognitive behavior therapy for schizophrenia. Schizophrenia Bulletin, 35, 857-858.

Chadwick, P. D. J. and Birchwood, M. (1994). The omnipotence of voices: a cognitive approach to auditory hallucinations. British Journal of Psychiatry, 164, 190-201.

David, A. S., Buchanan, A., Reed, A. and Almeida, O. (1992). The assessment of insight in psychosis. British Journal of Psychiatry, 161, 599-602.

Drake, R., Haddock, G., Tarrier, N., Bentall, R. and Lewis, S. (2007). The Psychotic Symptom Rating Scales (PSYRATS): their usefulness and properties in first episode psychosis. Schizophrenia Research, 89, 119-122.

Durham, R. C., Chambers, J. A., Power, K. G., Sharp, D. M., MacDonald, R. R., Major, K. A., Dow, M. G. and Gumley, A. (2005). Long-term outcome of cognitive behaviour therapy clinical trials in central Scotland. Health Technology Assessment, 9 (42). 
Farhall, J., Greenwood, K. M. and Jackson, H. J. (2007). Coping with hallucinated voices in schizophrenia: a review of self-initiated strategies and therapeutic interventions. Clinical Psychology Review, 27, 476-493.

Favrod, J., Vianin, P., Pomini, V. and Mast, F. W. (2006). A first step toward cognitive remediation of voices: a case study. Cognitive Behaviour Therapy, 35, 159-163.

Fowler, D., Garety, E. and Kuipers, E. (1995). Cognitive Behaviour Therapy for Psychosis. Chichester: Wiley.

Garety, P. A., Fowler, D., Kuipers, E., Freeman, D., Dunn, G., Bebbington, P., Hadley, C. and Jones, S. (1997). London-East Anglia randomised controlled trial of cognitive-behavioural therapy for psychosis: II. predictors of outcome. British Journal of Psychiatry, 171, 420426.

Garety, P. A., Fowler, D. and Kuipers, E. (2000). Cognitive-behavioral therapy for medicationresistant symptoms. Schizophrenia Bulletin, 26, 1-14.

Gaudiano, B. A. and Herbert, J. D. (2006). Acute treatment of inpatients with psychotic symptoms using Acceptance and Commitment Therapy: pilot results. Behaviour Research and Therapy, 44, 415-437.

Haddock, G., McCarron, J., Tarrier, N. and Faragher, E. B. (1999). Scales to measure dimensions of hallucinations and delusions: the Psychotic Symptom Rating Scales (PSYRATS). Psychological Medicine, 29, 879-889.

Haddock, G., Slade, P. D., Bentall, R. P., Reid, D. and Faragher, E. B. (1998). A comparison of the long-term effectiveness of distraction and focusing in the treatment of auditory hallucinations. British Journal of Medical Psychology, 71, 339-349.

Hayward, M., Overton, J., Dorey, T. and Denney, J. (2009). Relating therapy for people who hear voices: a case series. Clinical Psychology and Psychotherapy, 16, 216-227.

Kay, S., Opler, L. and Lindenmayer, J. P. (1987). The Positive and Negative Syndrome Scale (PANSS) for schizophrenia. Schizophrenia Bulletin, 13, 261-275.

Kay, S., Opler, L. and Lindenmayer, J. P. (1988). Reliability and validity of the positive and negative syndrome scale for schizophrenics. Psychiatry Research, 23, 99-110.

Kirkpatrick, B., Buchanan, R. W., Ross, D. E. and Carpenter, W. T. (2001). A separate disease within the syndrome of schizophrenia. Archives of General Psychiatry, 58, 165-171.

Liddle, P. F. (1987). The symptoms of chronic schizophrenia: a re-examination of the positive-negative dichotomy. British Journal of Psychiatry, 151, 145-151.

McGowan, J. F., Lavender, T. and Garety, P. A. (2005). Factors in outcome of cognitive-behavioural therapy for psychosis: users' and clinicians' views. Psychology and Psychotherapy: Theory, Research and Practice, 78, 513-529.

Morrison, A. P. and Renton, J. C. (2001). Cognitive therapy for auditory hallucinations: a theory-based approach. Cognitive and Behavioral Practice, 8, 147-160.

Morrison, A. P., Renton, J., Dunn, H., Williams, S. and Bentall, R. (2004). Cognitive Therapy for Psychosis: a formulation-based approach. London: Brunner-Routledge.

Naeem, F., Kingdon, D. and Turkington, D. (2008). Predictors of response to cognitive behavior therapy in the treatment of schizophrenia: a comparison of brief and standard interventions. Cognitive Therapy and Research, 32, 651-656.

National Institute for Clinical Excellence (2009). Schizophrenia: core interventions in the treatment and management of schizophrenia in primary and secondary care (update). London: Department of Health.

Rector, N. A., Beck, A. T. and Stolar, N. (2005). The negative symptoms of schizophrenia: a cognitive perspective. Canadian Journal of Psychiatry, 50, 247-257.

Shawyer, F., Farhall, J., Sims, E. and Copolov, D. (2005). Command hallucinations in psychosis: acceptance and disengagement as a focus of treatment. In M. Jackson and G. Murphy (Eds.), Theory and Practice in Contemporary Australian Cognitive and Behaviour Therapy: proceedings of the 
28th AACBT conference (pp. 5-14). Melbourne: Australian Association for Cognitive and Behaviour Therapy.

Tarrier, N. (1992). Management and modification of residual positive psychotic symptoms. In M. Birchwood and N. Tarrier (Eds.), Innovations in the Psychological Management of Schizophrenia. Chichester: Wiley.

Tarrier, N., Beckett, R., Harwood, S., Baker, A., Yusupoff, L. and Ugarteburu, I. (1993). A trial of two cognitive-behavioural methods of treating drug-resistant residual psychotic symptoms in schizophrenic patients. I: outcome. British Journal of Psychiatry, 162, 524-532.

Tarrier, N., Yusupoff, L., Kinney, C., McCarthy, E., Gledhill, A., Haddock, G. and Morris, J. (1998). Randomised controlled trial of intensive cognitive behaviour therapy for patients with chronic schizophrenia. British Medical Journal, 317, 303-307.

Tarrier, N., Kinney, C., McCarthy, E., Wittkowski, A., Yusupoff, L., Gledhill, A., Morris, J. and Humphreys, L. (2001). Are some types of psychotic symptoms more responsive to cognitivebehaviour therapy? Behavioural and Cognitive Psychotherapy, 29, 45-55.

Trower, P., Birchwood, M., Meaden, A., Byrne, S., Nelson, A. and Ross, K. (2004). Cognitive therapy for command hallucinations: randomised controlled trial. British Journal of Psychiatry, 184, 312 320 .

Turkington, D., Kingdon, D. and Turner, T. (2002). Effectiveness of a brief cognitive-behavioural therapy intervention in the treatment of schizophrenia. British Journal of Psychiatry, 180, 523-527.

Wykes, T., Steel, C., Everitt, B. and Tarrier, N. (2008). Cognitive behavior therapy for schizophrenia: effect sizes, clinical models, and methodological rigor. Schizophrenia Bulletin, 34, 523-537. 


\section{University Library}

\section{- M M N E R VA A gateway to Melbourne's research publications}

Minerva Access is the Institutional Repository of The University of Melbourne

Author/s:

Thomas, N;Rossell, S;Farhall, J;Shawyer, F;Castle, D

Title:

Cognitive Behavioural Therapy for Auditory Hallucinations: Effectiveness and Predictors of Outcome in a Specialist Clinic

Date:

2011-03-01

Citation:

Thomas, N., Rossell, S., Farhall, J., Shawyer, F. \& Castle, D. (2011). Cognitive Behavioural Therapy for Auditory Hallucinations: Effectiveness and Predictors of Outcome in a Specialist Clinic. BEHAVIOURAL AND COGNITIVE PSYCHOTHERAPY, 39 (2), pp.129-138. https:// doi.org/10.1017/S1352465810000548.

Publication Status:

Published

Persistent Link:

http://hdl.handle.net/11343/33024 